\title{
Bridge carrying robot using ZigBee carrying multiple sensor
}

\author{
M. Dheeraj Reddy \\ Department of Electronics and Communication Engineering, \\ Pydah College of Engineering \& Technology, Gambheeram Village, \\ Anandapuram Mandal, Visakhapatnam - 531 163, India \\ E-mail address: dheeraj8448@gmail.com
}

\begin{abstract}
MULTI-UTILITY VEHICLE is a vehicle which carries a folding bridge and multiple sensors. This vehicle is mainly used in war-field, it helps the soldiers to cross The un-even areas with the help of the bridge. It also helps other heavy vehicles to cross the disturbed path using its bridge. It is also equipped with multiple sensors like the fire detector, gas detector, light detector. This helps the soldiers to identify the different types of threats bearing poisonous gases, increase in temperature etc. from a longer distance. Those threats can be recognized by the soldiers with the help of buzzers equipped in the detector. As it is a remote vehicle which works on the wireless transmission of ZigBee, the light detector in the vehicle is used to automatically switch on the main lights when the visibility level starts decreasing due to sunlight. This project is already implemented in European countries during the First World War. It is an upcoming technology in our country. For solving the problem of the cooperation between multi-robots, a communication system based on the ZigBee network was designed. In the hardware of the robots, the ZigBee nodes are introduced. The multirobots form a star network by the ZigBee nodes, and they keep communication with each other by the central node. The ZigBee network has many characteristics such as low cost, low power consume, strong anti-jamming ability and real-time performance etc, it adapts to the application of intelligence. Experiments showed that the communication between the robots is reliable. This scheme is a good way for robots to complete complex task and improve working efficiency.
\end{abstract}

Keywords: ZigBee; Micro Controller; Light-dependent resistor (LDR)

\section{INTRODUCTION}

Mobile armoured forces are confronted with obstacles, manmade and natural feature's, that threaten forward progress, including craters, anti-tank ditches, blown bridges, railroad cuts, canals, rivers and ravines. The Armoured Vehicle Launched Bridge (AVLB) moves with the armoured force to provide an immediate solution to overcome the obstacles.

The Armoured Vehicle Launched Bridge (AVLB) is an aluminium alloy folding bridge carried by a tank chassis, the AVLB bridge is unfolded across the gap using torque provided by the launcher vehicle to actuate a motor inside the bridge structure. The power and traction of the vehicle also made it an excellent tank retriever, when not being used in its primary bridging role. The AVLB can launch its bridge without exposing the crew of two to enemy fire. After assault force vehicles have passed over the bridge, the AVLB can cross over, pick 
up the bridge on the far bank and continue along in support of assault forces. This vehicle is also equipped with multiple sensors like Gas, Light, Fire detectors.

This vehicle is also considered as a Multi Utility Vehicle because whenever there is no use of the bridge this vehicle is used to check the obstacle using the different types of sensors which are placed in it. As it is a remote vehicle it can also equipped with a wireless camera which helps us to control the vehicle and as well to check the surroundings of the vehicle. This vehicle is remote vehicle which works on the principles of ZIGBEE. The (ZIGBEE) is a technique which is used to transmit and receive the information through wireless means. The Zigbee is used to transfer the information over long distances. The average ranging distance of a Zigbee module is approximately $10 \mathrm{~km}$ in radius.

\section{METHODOLOGY}

\section{MICROCONTROLLER}

A microcontroller (also MCU or $\mu \mathrm{C}$ ) is a small computer on a single integrated circuit consisting of a relatively simple CPU combined with support functions such as a crystal oscillator, timers, and watchdog, serial and analog I/O etc. Program memory in the form of NOR flash or OTP ROM is also often included on chip, as well as a, typically small, $\mathrm{read} / \mathrm{write}$ memory.Microcontrollers are used in automatically controlled products and devices, such as automobile engine control systems, remote controls, office machines, appliances, power tools, and toys. By reducing the size and cost compared to a design that uses a separate microprocessor, memory, and input/output devices, microcontrollers make it economical to digitally control even more devices and processes.

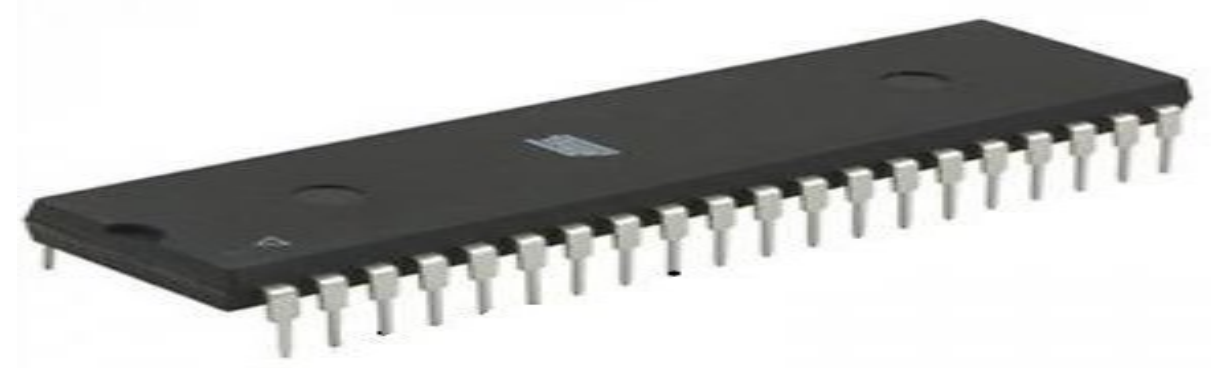

Micro Controller

The integrated circuit from an Intel 8742, an 8-bit microcontroller that includes a CPU running at $12 \mathrm{MHz}, 128$ bytes of RAM, 2048 bytes of EPROM, and I/O in the on chip.

\section{AT89C51 Micro Controller}

The AT89C51 is 80C51 microcontroller with $64 \mathrm{~KB}$ flash and $1024 \mathrm{~B}$ of data RAM. A key feature of the AT89C51 is its X2 mode option. The design engineer can choose to run the application with the conventional 80C51 clock rate (12 clocks per machine cycle) or select the $\mathrm{X} 2$ mode (six clocks per machine cycle) to achieve twice the throughput at the same clock frequency. Another way to benefit from this feature is to keep the same performance by reducing the clock frequency by half, thus dramatically reducing the EMI. 


\section{Block diagram}

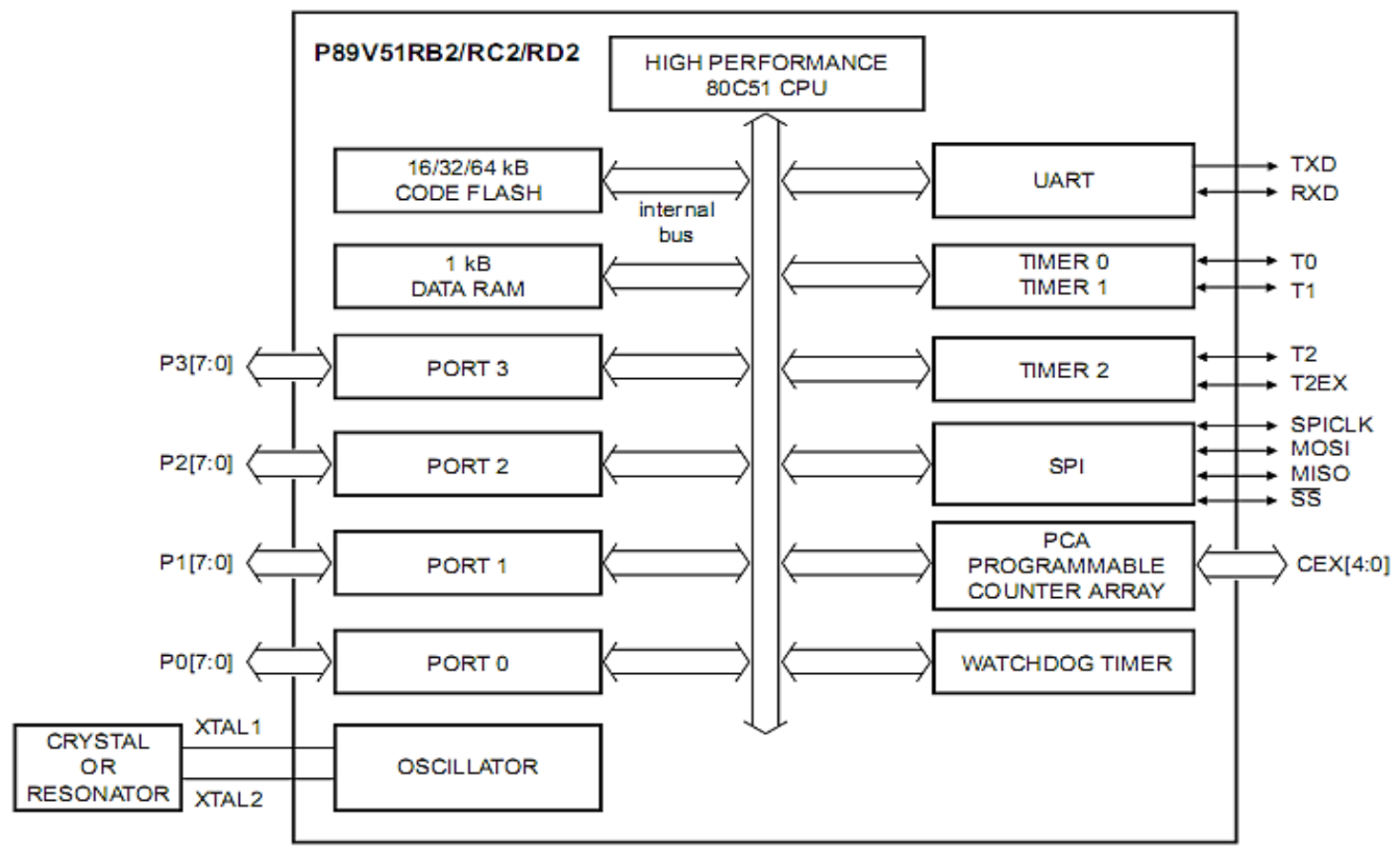

Microcontroller AT89C51

\section{PIN Configuration}

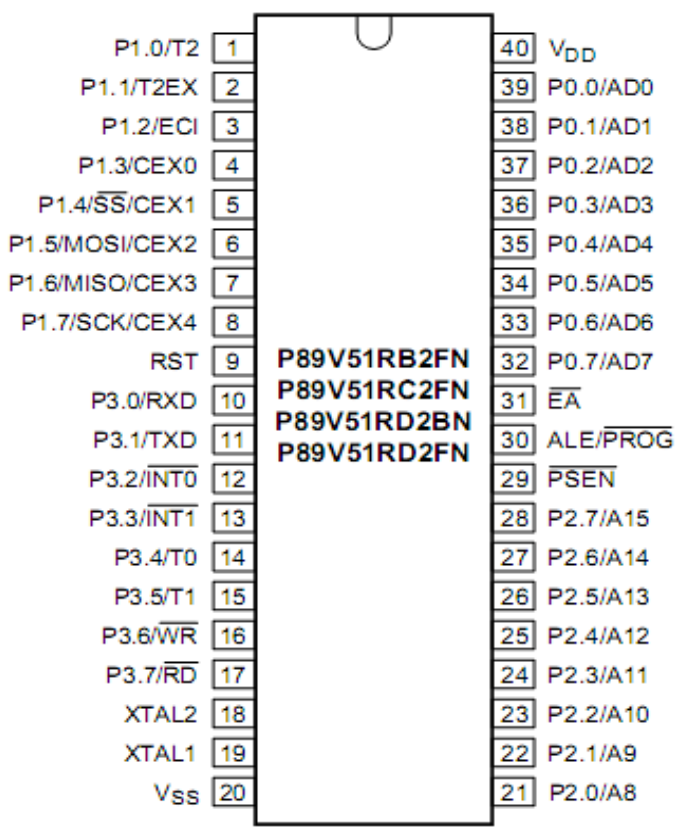

PIN Configuration of AT89C51 


\section{Flash Program Memory Bank Selection}

There are two internal flash memory blocks in the device. Block 0 has 16/32/64 kb and is Organized as 128/256/512 sectors, each sector consists of $128 \mathrm{~B}$. Block 1 contains the IAP/ISP routines and may be enabled such that it overlays the first $8 \mathrm{kB}$ of the user code memory. The overlay function is controlled by the combination of the Software Reset Bit (SWR) at FCF.1 and the Bank Select Bit (BSEL) at FCF.0.

The combination of these bits and the memory source used for instructions is shown below. Access to the IAP routines in block 1 may be enabled by clearing the BSEL bit (FCF.0), provided that the SWR bit (FCF.1) is cleared. Following a power-on sequence, the boot code is automatically executed and attempts to auto baud to a host. If no auto baud occurs within approximately $400 \mathrm{~ms}$ and the Soft ICE flag is not set, control will be passed to the user code.

A software reset is used to accomplish this control transfer and as a result the SWR bit will remain set. Therefore the user's code will need to clear the SWR bit in order to access the IAP routines in block 1 . However, caution must be taken when dynamically changing the BSEL bit

\section{Data RAM Memory}

The data RAM has $1024 \mathrm{~B}$ of internal memory. The device can also address up to $64 \mathrm{kB}$ for external data memory.

\section{Expanded Data RAM Addressing}

The device has four sections of internal data memory:

1. The lower $128 \mathrm{~B}$ of RAM $(00 \mathrm{H}$ to $7 \mathrm{FH})$ is directly and indirectly addressable.

2. The higher $128 \mathrm{~B}$ of RAM $(80 \mathrm{H}$ to $\mathrm{FFH})$ is indirectly addressable.

3 . The special function registers $(80 \mathrm{H}$ to $\mathrm{FFH})$ are directly addressable only.

4. The expanded RAM of $768 \mathrm{~B}(00 \mathrm{H}$ to $2 \mathrm{FFH})$ is indirectly addressable by the move external instruction (MOVX) and clearing the EXTRAM bit.

Since the upper 128 B occupy the same addresses as the SFRs, the RAM must be accessed indirectly. The RAM and SFRs space are physically separate even though they have the same addresses.

\section{Flash Organization}

TheAT89C51 program memory consists of a $64 \mathrm{kB}$ block. ISP capability, in a second 8 $\mathrm{kB}$ block, is provided to allow the user code to be programmed in-circuit through the serial port. There are three methods of erasing or programming of the flash memory that may be used.

First, the flash may be programmed or erased in the end-user application by calling lowlevel routines through a common entry point (IAP). Secondly the on-chip ISP boot loader may be invoked.

This ISP boot loader will, in turn, call low-level routines through the same common entry point that can be used by the end-user application. Third, the flash may be programmed or erased using the parallel method by using a commercially available EPROM programmer which supports this device. 

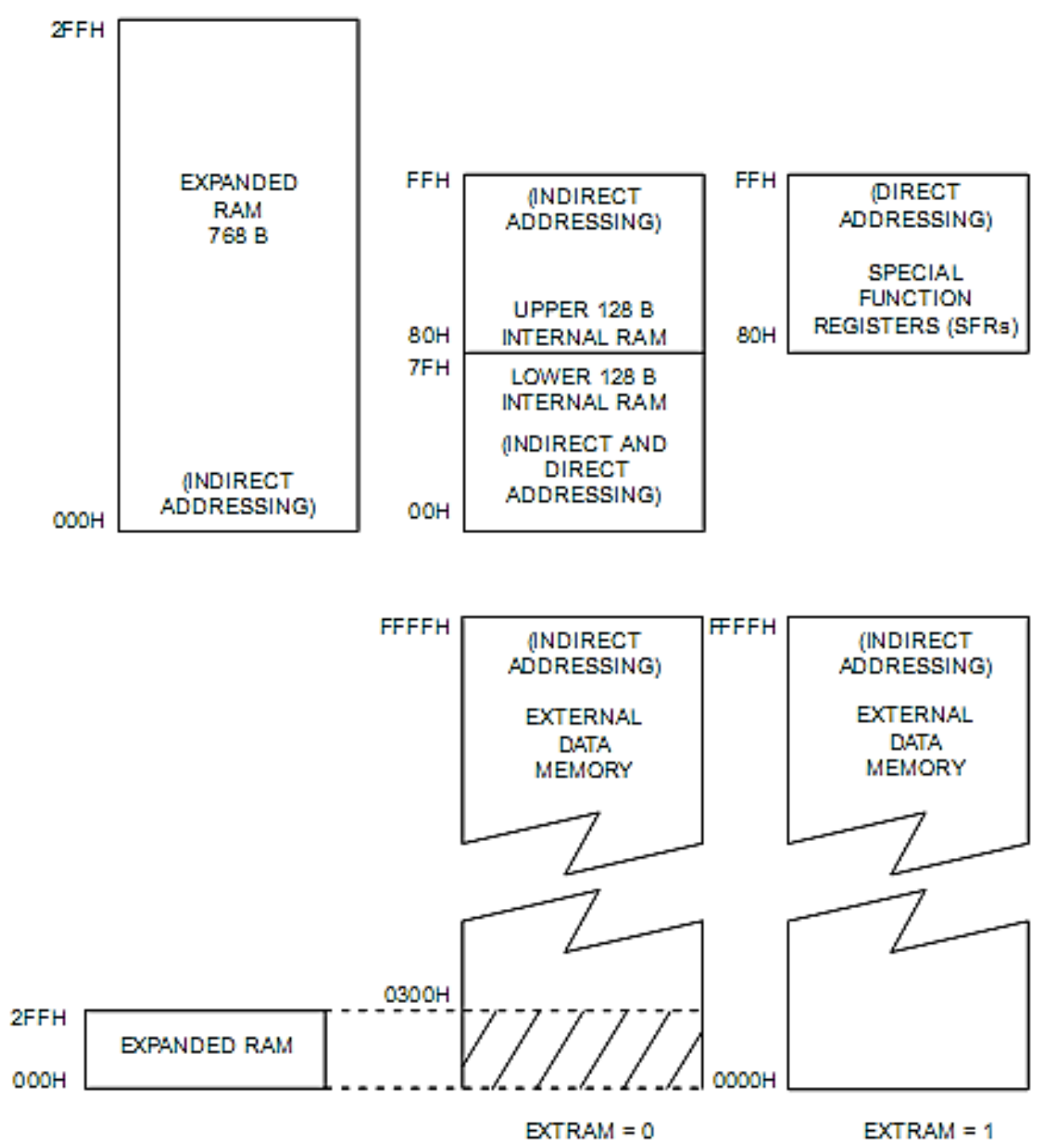

Internal and External data memory structure

The AT89C51 program memory consists of a $64 \mathrm{kB}$ block. ISP capability, in a second 8 $\mathrm{kB}$ block, is provided to allow the user code to be programmed in-circuit through the serial port. There are three methods of erasing or programming of the flash memory that may be used. First, the flash may be programmed or erased in the end-user application by calling lowlevel routines through a common entry point (IAP). Secondly the on-chip ISP boot loader may be invoked. This ISP boot loader will, in turn, call low-level routines through the same common entry point that can be used by the end-user application. Third, the flash may be programmed or erased using the parallel method by using a commercially available EPROM programmer which supports this device.

\section{Interrupts}

It is mandatory that microcontrollers provide real time response to events in the embedded system they are controlling. When certain events occur an interrupt system can signal the processor to suspend processing the current instruction sequence and to begin an interrupt service routine (ISR). The ISR will perform any processing required based on the source of the interrupt before returning to the original instruction sequence. 


\begin{tabular}{|c|c|c|c|c|c|c|}
\hline Description & Interrupt flag & Vector address & $\begin{array}{l}\text { Interrupt } \\
\text { enable }\end{array}$ & $\begin{array}{l}\text { Interrupt } \\
\text { priority }\end{array}$ & $\begin{array}{l}\text { Service } \\
\text { priority }\end{array}$ & $\begin{array}{l}\text { Wake-up } \\
\text { power-down }\end{array}$ \\
\hline Ext. Int0 & IEO & $0003 \mathrm{H}$ & EXO & $\mathrm{PXO} / \mathrm{H}$ & 1 (highest) & yes \\
\hline Brownout & - & 004BH & EBO & $\mathrm{PBO} / \mathrm{H}$ & 2 & no \\
\hline TO & TF0 & OOOBH & ETO & PTO/H & 3 & no \\
\hline Ext. Int1 & IE1 & $0013 \mathrm{H}$ & EX1 & $\mathrm{PX} 1 / \mathrm{H}$ & 4 & yes \\
\hline T1 & TF1 & $001 \mathrm{BH}$ & ET1 & $\mathrm{PT} 1 / \mathrm{H}$ & 5 & no \\
\hline $\mathrm{PCA}$ & CF/CCFn & $0033 \mathrm{H}$ & EC & $\mathrm{PPCH}$ & 6 & no \\
\hline UART/SPI & TI/RI/SPIF & $0023 \mathrm{H}$ & ES & $\mathrm{PS} / \mathrm{H}$ & 7 & no \\
\hline $\mathrm{T} 2$ & TF2, EXF2 & 002BH & ET2 & $\mathrm{PT} 2 / \mathrm{H}$ & 8 & no \\
\hline
\end{tabular}

Interrupt Priority and Polling Sequence

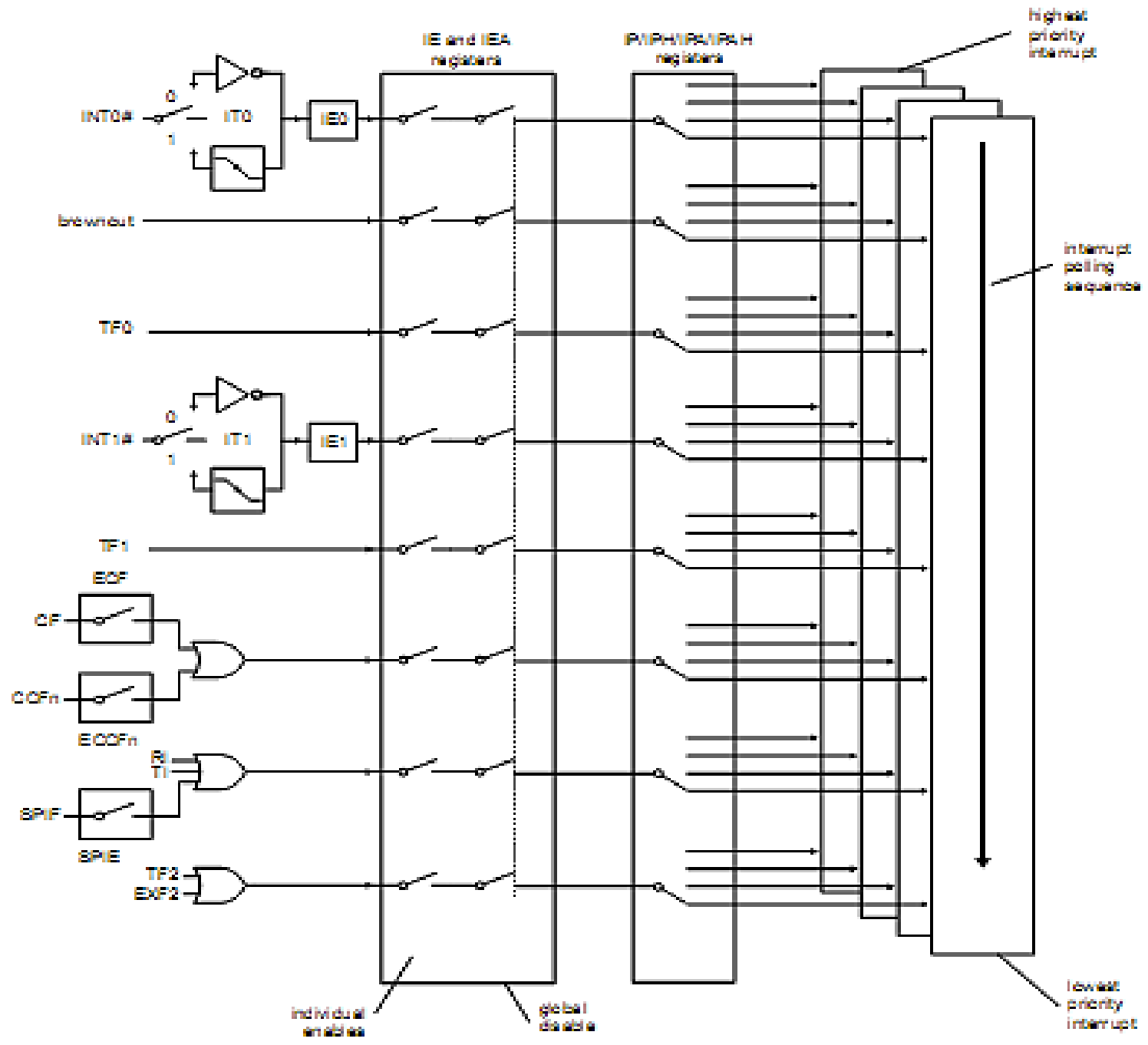

Interrupt Structure 
Possible interrupt sources are device dependent, and often include events such as an internal timer overflow, completing an analogy to digital conversion, a logic level change on an input such as from a button being pressed, and data received on a communication link. Where power consumption is important as in battery operated devices, interrupts may also wake a microcontroller from a low power sleep state where the processor is halted until required to do something by a peripheral event.

\section{ZIGBEE}

ZigBee is a low data rate, two-way standard for home automation and data. The standard specification for up to 254 nodes including one master, managed from a networks such as turning lights on, setting the home security system, or starting the VCR. With ZigBee all these tasks can be done from anywhere in the home at the touch of a button. ZigBee also allows for dial-in access via the Internet for automation control.

The ZigBee standard uses small very low power devices to connect to form a wireless control web. A ZigBee network is capable of supporting up to 254 client nodes plus one full functional device (master). ZigBee protocol is optimized for very long battery life measured in months to years from inexpensive, off the shelf non rechargeable batteries, and can control lighting, air conditioning and smoke and fire alarms, and other security devices. The standard supports $2.4 \mathrm{GHz}$ (Worldwide), $868 \mathrm{MHz}$ (Europe) and 915(America) unlicensed radio bands with range up to 75 meters.

\section{IEEE 802.15.4}

IEEE 802.15.4 is a standard which specifies the physical layer and medium access control for low-rate wireless personal area networks (LR-WPAN's).This standard was chartered to investigate a low data rate solution with multi-month to multi-year battery life and very low complexity. It is operating in an unlicensed, international frequency band. Potential applications are sensors, interactive toys, smart badges, remote controls, and home automation. 802.15.4 Is part of the 802.15 wireless personal-area network effort at the IEEE. It is a simple packet-based radio protocol aimed at very low-cost, battery-operated widgets and sensors (whose batteries last years, not hours) that can intercommunicate and send lowbandwidth data to a centralized device.

\section{Channel access, addressing}

Two channel-access mechanisms are implemented in 802.15.4. For a none" beacon network, a standard CSMA-CA (carrier-sense medium-access with collision avoidance) communicates with positive acknowledgement for successfully received packets. In a beaconenabled network, a super frame structure is used to control channel access. The super frame is set up by the network coordinator to transmit beacons at predetermined intervals (multiples of $15.38 \mathrm{~ms}$, up to $252 \mathrm{~s}$ ) and provides 16 equal-width time slots between beacons for contentionfree channel access in each time slot. The structure guarantees dedicated bandwidth and low latency. Channel access in each time slot is contention-based. However, the network coordinator can dedicate up to seven guaranteed time slots per beacon interval for quality of service. Device addresses employ 64-bit IEEE and optional 16-bit short addressing. The address field within the MAC can contain both source and destination address information (needed for peer-to-peer operation). This dual address information is used in mesh networks to prevent a single point of failure within the network. 


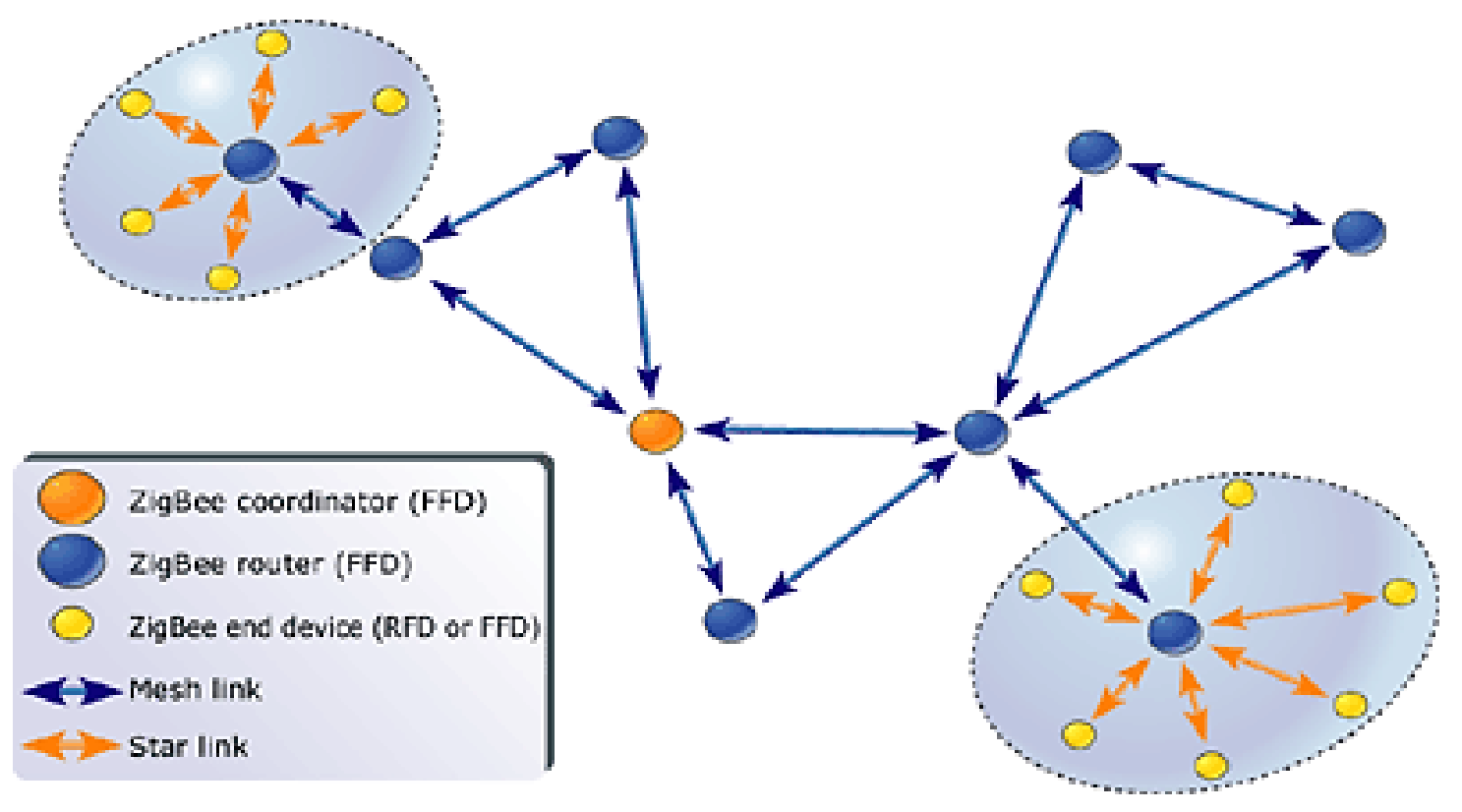

ZigBee network model

\section{Secure Connections}

ZigBee leverages the security model of the IEEE 802.15.4 MAC sub layer which specifies four security services:

- access control - the device maintains a list of trusted devices within the network

- Data encryption, which uses symmetric key 128-bit advanced encryption standard(AES).

- frame integrity to protect data from being modified by parties without cryptographic keys

- Sequential freshness to reject data frames that have been replayed - the network controller compares the freshness value with the last known value from the device and rejects it if the freshness value has not been updated to a new value

The actual security implementation is specified by the implementer using a standardized toolbox of ZigBee security software.

\section{Zigbee Advantages}

In all of its uses, Zigbee offers four inherent, beneficial characteristics:

- Low cost

- Range and obstruction issues avoidance

- Multi-source products

- Low power consumption 


\section{Design and Implementation}

\section{Motors}

From the start, DC motors seem quite simple. Apply a voltage to both terminals, and we it spins. But what if you want to control which direction the motor spins? Correct, you reverse the wires. Now what if you want the motor to spin at half that speed? You would use less voltage. But how would you get a robot to do those things autonomously? How would you know what voltage a motor should get? Why not $50 \mathrm{~V}$ instead of $12 \mathrm{~V}$ ? What about motor overheating? Operating motors can be much more complicated than you think.

\section{Voltage}

We probably know that DC motors are non-polarized - meaning that you can reverse voltage without any bad things happening. Typical DC motors are rated from about $6 \mathrm{~V}-12 \mathrm{~V}$. The larger ones are often $24 \mathrm{~V}$ or more. But for the purposes of a robot, you probably will stay in the $6 \mathrm{~V}-12 \mathrm{~V}$ range. So why do motors operate at different voltages? As we all know (or should), voltage is directly related to motor torque. Greater the voltage higher the torque. But don't go running your motor at $100 \mathrm{~V}$ cause that's just not nice. A DC motor is rated at the voltage it is most efficient at running. If you apply too few volts, it just won't work. If you apply too much, it will overheat and the coils will melt. So the general rule is, try to apply as close to the rated voltage of the motor as you can. Also, although a $24 \mathrm{~V}$ motor might be stronger, do you really want your robot to carry a $24 \mathrm{~V}$ battery (which is heavier and bigger) around? My recommendation is do not surpass $12 \mathrm{~V}$ motors unless you really need the torque.

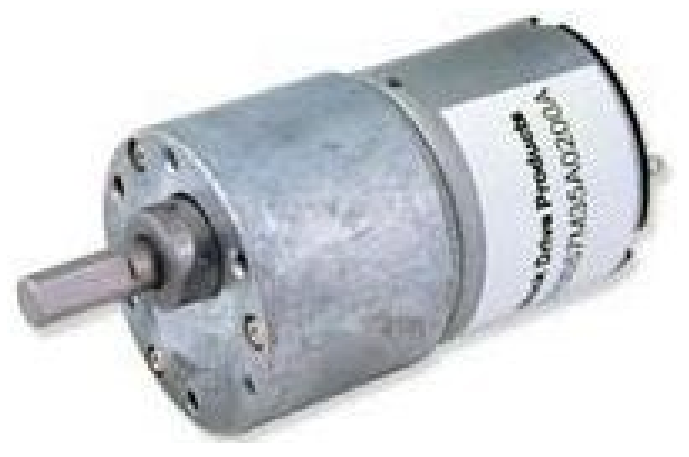

DC Motor

\section{L293D Dual H-Bridges Motor Driver}

L293D is a dual H-Bridge motor driver, So with one IC we can interface two DC motors which can be controlled in both clockwise and counter clockwise direction and if you have motor with fix direction of motion the you can make use of all the four I/Os to connect up to four DC motors. L293D has output current of $600 \mathrm{~mA}$ and peak output current of 1.2A as per the truth mentioned in the image above its fairly simple to program the microcontroller. It's also clear from the truth table of BJT circuit and L293D the programming will be same for both of them, just keeping in mind the allowed combinations of $\mathrm{A}$ and We will discuss about programming in $\mathrm{C}$ as well as assembly for running motor with the help of a 
microcontroller. B. per channel. Moreover for protection of circuit from back EMF output diodes are included within the IC. The output supply (VCC2) has a wide range from $4.5 \mathrm{~V}$ to $36 \mathrm{~V}$, which has made L293D a best choice for DC motor driver. As you can see in the circuit, three pins are needed for interfacing a DC motor (A, B, Enable). If you want the o/p to be enabled completely then you can connect Enable to VCC and only 2 pins needed from controller to make the motor work.

\section{MAJOR COMPONENTS IN SENSORS}

\section{Thermistor}

A thermistor is a type of resistor whose resistance varies significantly with temperature, more so than in standard resistors. The word is a portmanteau of thermal and resistor. Thermistors are widely used as inrush current limiters, temperature sensors, self-resetting overcurrent protectors, and self-regulating heating elements. Thermistors differ from resistance temperature detectors (RTD) in that the material used in a thermistor is generally a ceramic or polymer, while RTDs use pure metals. The temperature response is also different; RTDs are useful over larger temperature ranges, while thermistors typically achieve a higher precision within a limited temperature range, typically $-90{ }^{\circ} \mathrm{C}$ to $130{ }^{\circ} \mathrm{C}$.

It is the major component in the Fire detecting sensor.

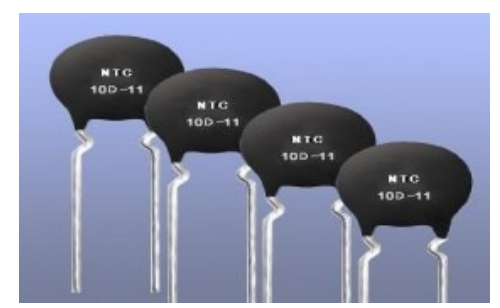

Thermistor

\section{Photo resistor}

A photo resistor or light-dependent resistor (LDR) or photocell is a light-controlled variable resistor. The resistance of a photo resistor decreases with increasing incident light intensity; in other words, it exhibits photoconductivity. A photo resistor can be applied in light-sensitive detector circuits, and light- and dark-activated switching circuits. A photo resistor is made of a high resistance semiconductor.

In the dark, a photo resistor can have a resistance as high as a few Mega ohms (M $\Omega)$, while in the light, a photo resistor can have a resistance as low as a few hundred ohms. If incident light on a photo resistor exceeds a certain frequency, photons absorbed by the semiconductor give bound electrons enough energy to jump into the conduction band. The resulting free electrons (and their hole partners) conduct electricity, thereby lowering resistance.

The resistance range and sensitivity of a photo resistor can substantially differ among dissimilar devices. Moreover, unique photo resistors may react substantially differently to photons within certain wavelength bands. A photoelectric device can be either intrinsic or extrinsic.

An intrinsic semiconductor has its own charge carriers and is not an efficient semiconductor, for example, silicon. In intrinsic devices the only available electrons are in the 
valence band, and hence the photon must have enough energy to excite the electron across the entire band gap. Extrinsic devices have impurities, also called dopants, added whose ground state energy is closer to the conduction band; since the electrons do not have as far to jump, lower energy photons (that is, longer wavelengths and lower frequencies) are sufficient to trigger the device. If a sample of silicon has some of its atoms replaced by phosphorus atoms (impurities), there will be extra electrons available for conduction. This is an example of an extrinsic.

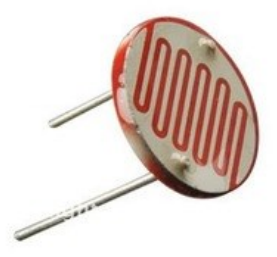

LDR

\section{Transmitter}

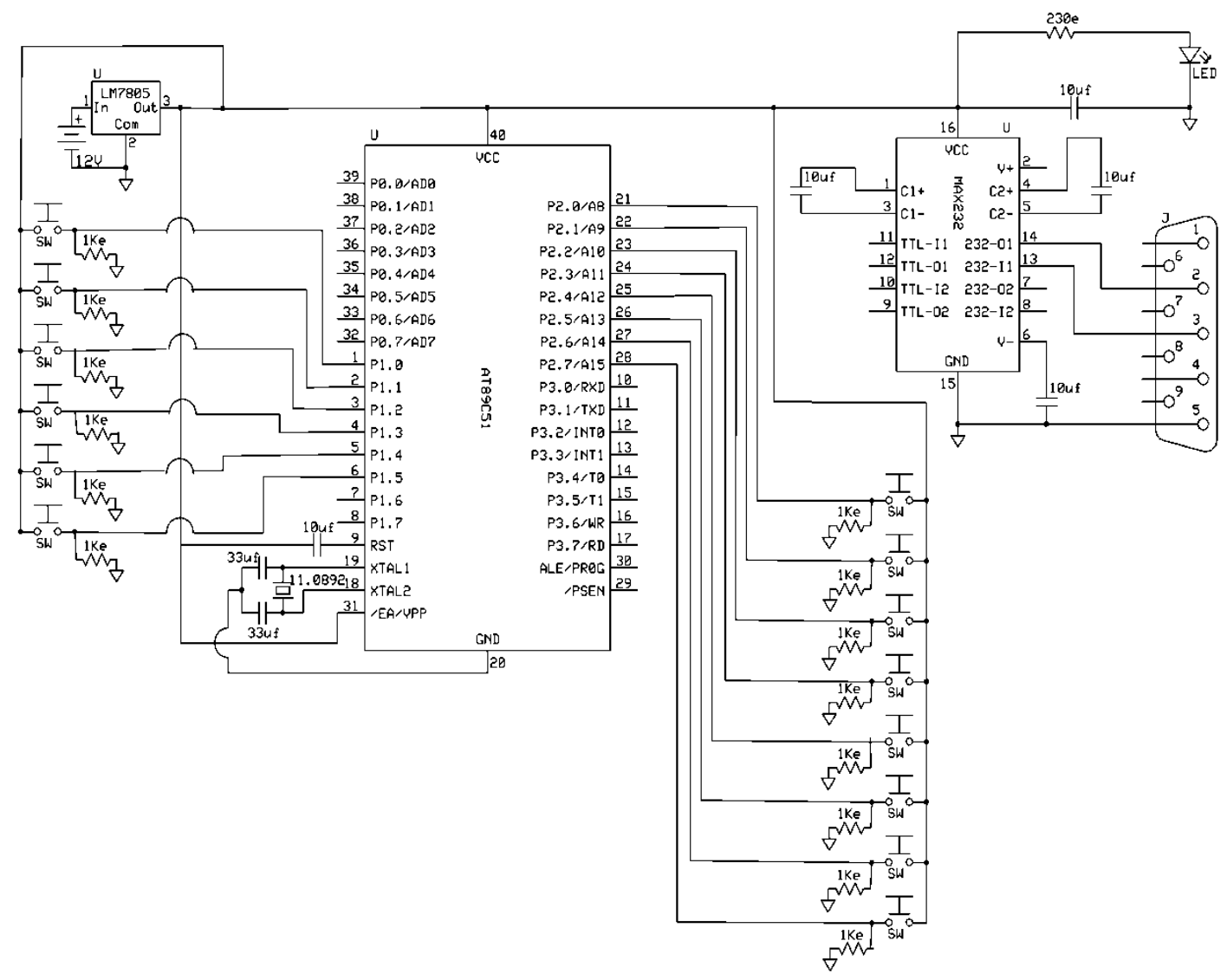




\section{Receiver}

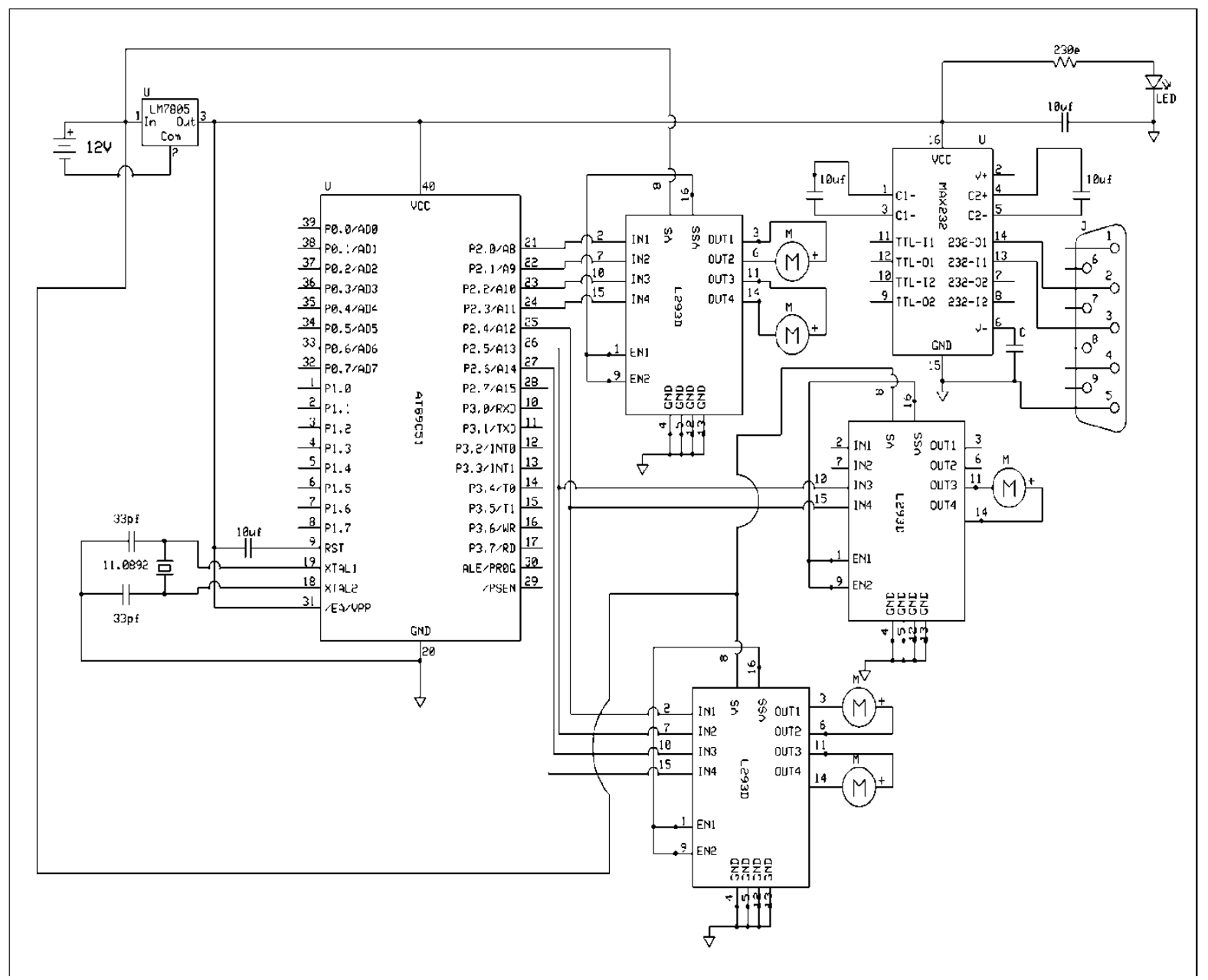

\begin{tabular}{|c|c|}
\hline \multicolumn{2}{|c|}{ Specifications } \\
\hline Weight (Estimated) & $3 \mathrm{~kg}$ \\
\hline Bridge dimension & length $70 \mathrm{~cm}$ \\
\hline Motor Rpm & $100 \mathrm{rpm}$ \\
\hline Zigbee range & $5 \mathrm{kms}$ in radius \\
\hline Frequency of Operation & $2.5 \mathrm{Ghz}$ \\
\hline Length of vehicle & $28 \mathrm{cms}$ \\
\hline Sensors & Metal, Light, Fire \\
\hline
\end{tabular}




\section{CONCLUSIONS}

The overall per cent change in fatigue life by increasing capacity from MLC 70 to MLC 80 depended on the model used and ranges from 50-80 percent. The stress-life approach provides a more conservative estimate of the bridge's life than does the strain-life approach. The Hinge and Top Chord are more critical than the Bottom Chord and Splice Plate, in terms of determining the fatigue life of the AVLB. Depending on the critical crack size of the component, a fracture analysis may be a more or less conservative estimate of fatigue life for the AVLB. The fatigue lives of the Hinge and Top Chord are affected substantially by the presence of a crack. Because of this, more focus should be placed on these components during bridge inspections. The results of this study constitutes the first model testing on an AVLB. From the testing we were able to identify the actual bending modes with different boundary conditions. The results indicates that the AVLB behaves as four losley coupled girders. This information is helpful in system design, namely a decision is made that the girders should be tested on individual basis. This test also provides information on the frequency bandwidth of interest and the necessary excitation level. This test alsovalidate the model provided by the result.
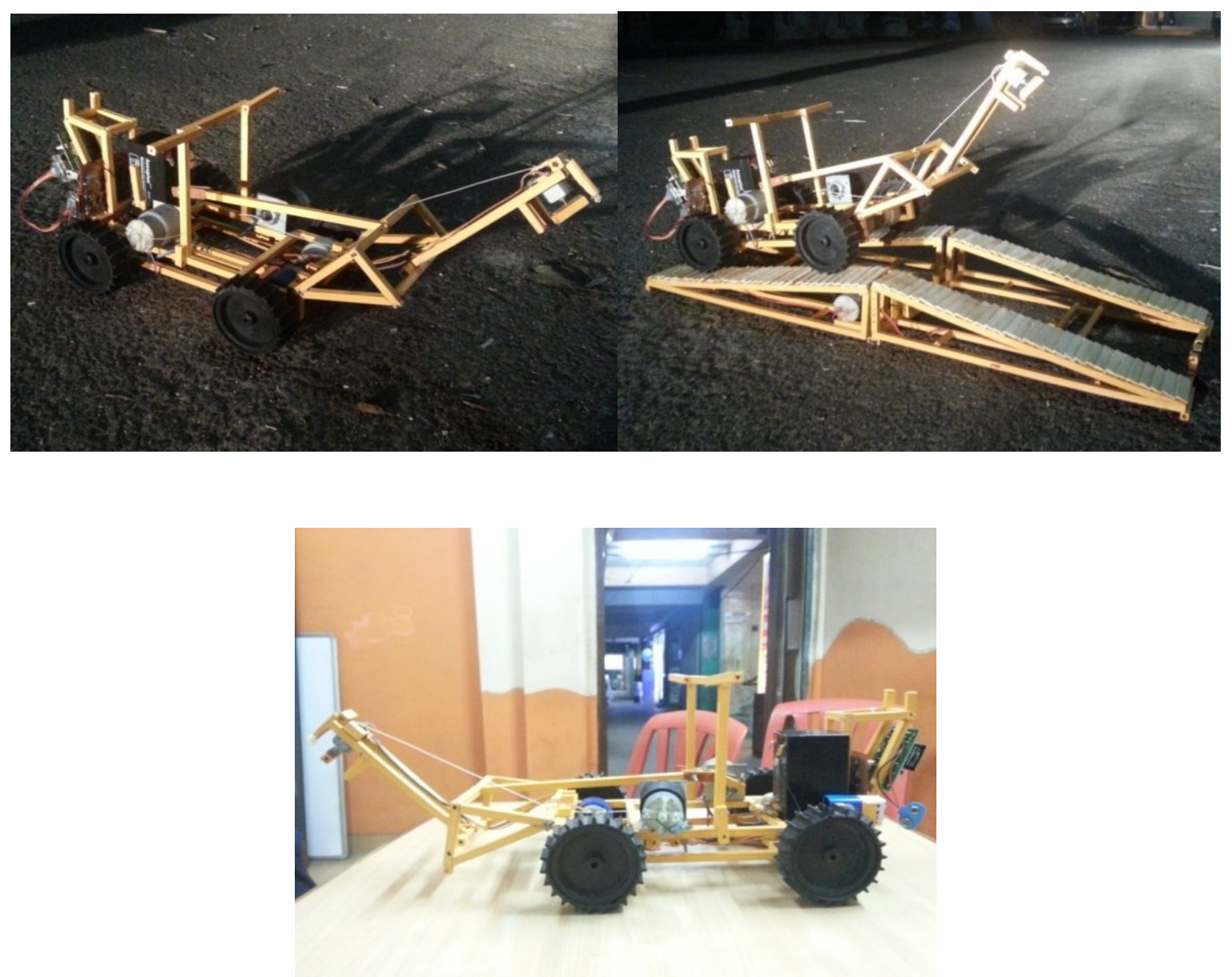

Pictures of the bridge carrying robot using ZigBee 


\section{Acknowledgement}

I am thankful to my Parents, my Father Mr. M. Ramesh Reddy\& my Mother M. Sujatha without whose support I couldn't have done anything and Mr. H. Rahaman Khan of Department of Biotechnology, GITAM Institute of Science, GITAM University, Visakhapatnam for preparing this paper.

\section{References}

[1] R. C. Luo, K. L. Su, S. H. Shen, et al., Proceedings of the IEEE 91(3) (2003)371-382.

[2] J.-H. Lee, H. Hashimoto, IEEE/ASME Transactions on Mechatronics 50(5) (2003) 890-902.

[3] S. Baeg, J. Park, J. Koh, et al., "Building a Smart Home Environment for Service Robots Based on RFID and Sensor Network," Proceedings of International Conference on Control, Automation and Systems, Seoul, 17-20 October 2007, pp. 1078-1082.

[4] L. Liang, L. Huang, X. Jiang, et al., "Design and Implementation of Wireless SmartHome Sensor Network Based on ZigBee Protocol," Proceedings of International Conference on Communications, Circuits and Systems, Xia- men, 25-27 May 2008, pp. 434-438.

[5] C. Suh, Y. Ko, IEEE Transactions on Consumer Electronics 54(3) (2008) 1177-1184.

[6] W. Yu, J. Lee, Y. Ha, et al., IEEE Transaction Automation Science and Engineering 6(4) (2009) 633-640.

[7] W. Lee, J. Kim, J. Kang, Sensors 10(9) (2010) 8663-8682.

[8] F. Zhou, G. Tian, Y. Xue, et al., Journal of Computational Information Systems 7(11) (2011) 3779-3786.

[9] F. Lu, G. Tian, F. Zhou, Intelligent Control and Automation 3(1) (2012) 90-97. 